\title{
Dynamique de la végétation ligneuse des espaces sylvo-pastoraux villageois mis en défens dans le Sud du Bassin arachidier au Sénégal
}

Marcel BADJI ${ }^{1}$

Diaminatou SANOGO ${ }^{2}$

Léonard Elie AKPO ${ }^{1}$

1 Université Cheikh Anta Diop Laboratoire d'écologie végétale et d'écohydrologie

(Ucad/Fst)

BP 5005, Dakar

Sénégal

2 Isra

Centre national de recherche

forestière (Cnrf) BP 2312, Dakar Sénégal

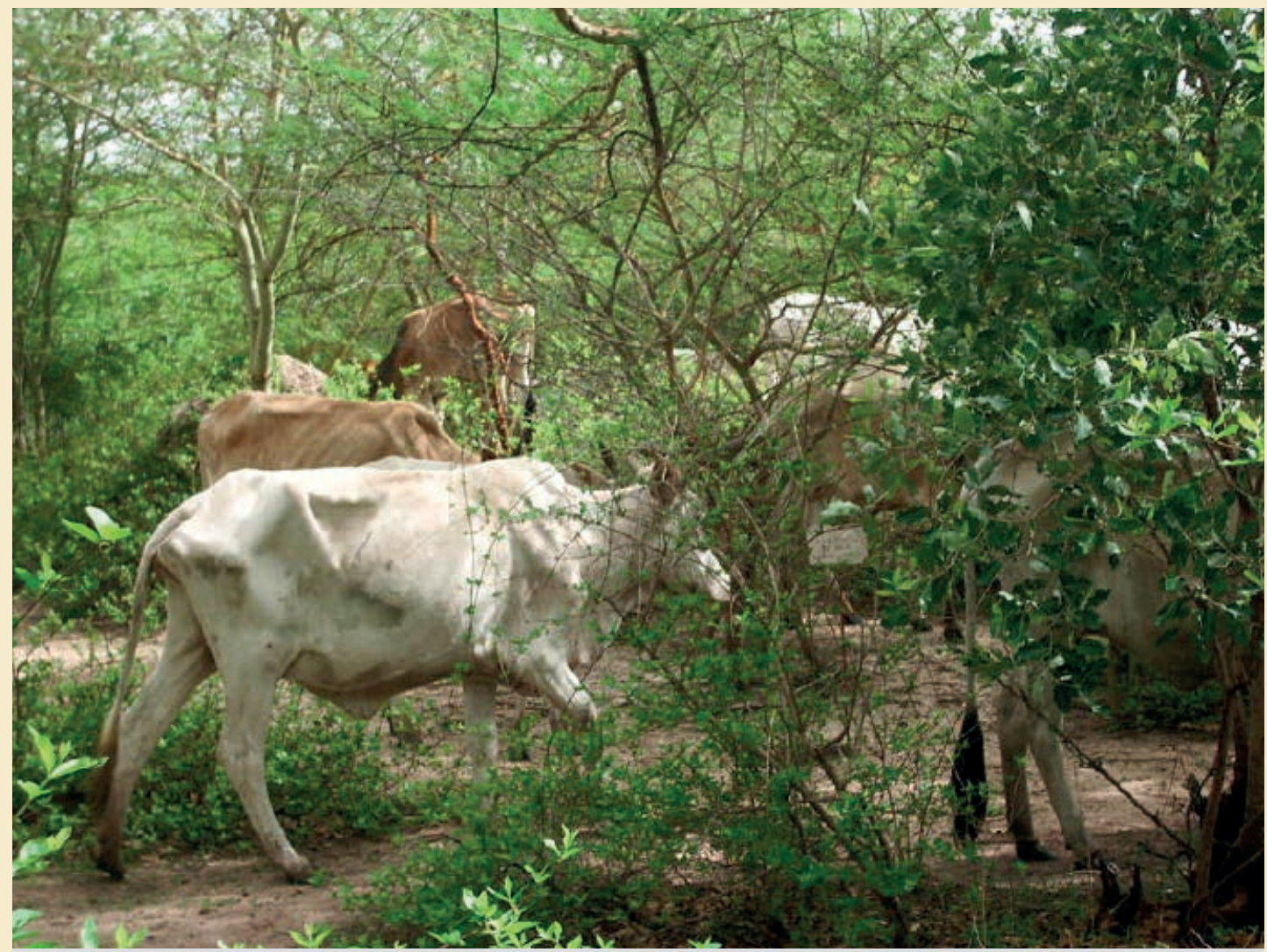

Photo 1.

Troupeau de bœufs pâturant dans la mise en défens.

Photo M. Badji. 


\section{RÉSUMÉ}

\section{DYNAMIQUE DE LA VÉGÉTATION LIGNEUSE DES ESPACES SYLVO- PASTORAUX VILLAGEOIS MIS EN DÉFENS DANS LE SUD DU BASSIN ARACHIDIER AU SÉNÉGAL}

Au Sénégal, dans le bassin arachidier en particulier, les espaces sylvo-pastoraux intervillageois sont surexploités et menacés de disparition à cause de l'extension des terres de culture et des coupes incontrôlées de ligneux pour le bois de chauffe et le charbon de bois. Face à la menace de disparition de ces espaces, les populations environnantes ont entrepris des actions de conservation et de réhabilitation de ces espaces par la mise en défens. Cette étude évalue le processus de reconstitution de la végétation des espaces sylvo-pastoraux intervillageois suite à leur mise en défens. L'étude a été menée dans le Sud du bassin arachidier, dans la région de Kaolack, au sein des sites mis en défens à des âges échelonnés : un an, cinq ans et douze ans. Les résultats d'inventaire montrent une faible diversité spécifique de vingt-sept espèces distinctes dans la mise en défens d'une année par rapport aux deux autres détenant chacune une cinquantaine d'espèces différentes. L'analyse de variance des paramètres structuraux indique une différence significative entre le diamètre moyen et la hauteur des arbres en fonction de l'âge des trois types de peuplements. Pour les mises en défens de cinq ans, la fréquence diamétrale des arbres répond à une distribution en " $L$ » décroissante, traduisant une dynamique régulière d'un jeune peuplement, alors que, pour celles de douze ans, il s'agit d'une distribution "en cloche " caractéristique de peuplements arborés devant atteindre un état d'équilibre. L'ensemble des mises en défens présente une bonne capacité de régénération ouvrant de bonnes perspectives pour la restauration des formations naturelles dégradées.

Mots-clés : mise en défens, espaces sylvo-pastoraux, caractéristiques structurales, régénération, communautés villageoises, Sénégal.

\section{ABSTRACT}

\section{DYNAMICS OF WOODY VEGETATION IN VILLAGE WOODLAND AND GRAZING RESERVES IN SOUTH SENEGAL'S GROUNDNUT BASIN}

In Senegal, and especially in the groundnut basins, woodlands and pastures lying between villages are being overexploited and are at risk of disappearing with the extension of croplands and uncontrolled wood-cutting for firewood and charcoal. Local populations have been attempting to counter the risk by restricting access to these lands to promote conservation and land rehabilitation. This study assesses the process of reconstitution of the vegetation in the village woodland and grazing reserves created. It was conducted in the southern groundnut basin in sites where reserves have been in place for one, five and twelve years. The results of the inventory show lower species diversity in the one year-old stands, with 27 distinct species, than in the other two age categories, where some 50 different species were found. The analysis of variance of the structural parameters shows a significant difference in the average diameter and height of trees according to the age of the three stand types. After five years, the diameter curve for the trees is L-shaped, reflecting the regular dynamics of a young tree stand, while the curve after twelve years has the characteristic bell shape of woodlands reaching a state of equilibrium. Regeneration capacities are high in all of the reserved lands, indicating good prospects for the restoration of these degraded natural woodlands.

Keywords: reserved lands, woodland and grazing areas, structural characteristics, regeneration, village communities, Senegal.

\section{RESUMEN}

\author{
DINÁMICA DE LA VEGETACIÓN LEÑOSA \\ DE LOS ESPACIOS SILVOPASTORILES \\ COMUNALES VEDADOS EN EL SUR \\ DE LA CUENCA MANISERA DE SENEGAL
}

En Senegal, concretamente en la cuenca manisera, los espacios silvopastoriles intercomunales están sobreexplotados y amenazados de desaparición por la extensión de tierras de cultivo y las talas incontroladas para leña y carbón. Ante la amenaza de desaparición de estos espacios, las poblaciones contiguas han acometido acciones de conservación y rehabilitación de dichos espacios mediante la aplicación de una veda. Este estudio evalúa el proceso de reconstitución de la vegetación de los espacios silvopastoriles intercomunales a raíz de su protección. El estudio se realizó en el sur de la cuenca manisera, en la región de Kaolack, dentro de los sitios vedados y escalonando las edades: un año, cinco años y doce años. Los resultados del inventario muestran una baja diversidad específica, veintisiete especies distintas, en la veda de un año frente a las dos otras categorías que poseen unas cincuenta especies diferentes cada una. El análisis de varianza de los parámetros estructurales indica una diferencia significativa entre el diámetro promedio y la altura de los árboles según la edad en los tres tipos de rodales. En las vedas de cinco años, la frecuencia diamétrica de los árboles responde a una distribución en "L" decreciente que refleja una dinámica regular de un rodal joven, mientras que en las de doce años se trata de una distribución "en campana”, característica de rodales arbolados que deben alcanzar un estado de equilibrio. El conjunto de áreas vedadas presenta una buena capacidad de regeneración que abre buenas perspectivas para la restauración de formaciones naturales degradadas.

Palabras clave: aplicación de una veda, espacios silvopastoriles, características estructurales, regeneración, comunidades aldeanas, Senegal. 


\section{Introduction}

Dans le bassin arachidier, les espaces sylvo-pastoraux constituent à la fois l'espace pastoral (photo 1), l'espace de cueillette, celui de collecte du bois pour les usages domestiques. Cependant, ces espaces subissent une réduction des boisements du fait de l'augmentation massive des superficies emblavées, d'une nette intensification des prélèvements des produits de cueillette, d'une coupe intensive et incontrôlée du bois (photo 2) et d'une pression accrue sur des ressources pastorales. Cela se traduit par la régression de la couverture végétale (GROUZIS, 1988), par la dégradation de l'environnement et par la baisse des rendements, d'où la nécessité d'une gestion durable (BAZILE, 1998). Face à cette dégradation, les populations tentent de préserver et de réhabiliter ces espaces par la mise en défens. Par définition, la mise en défens est une technique qui consiste à mettre au repos, par des rotations périodiques, des surfaces dégradées afin d'y favoriser la régénération des couvertures végétale et pédologique. L'enjeu le plus important de la mise en défens revient au rôle de la végétation dans la lutte contre les érosions hydrique et éolienne qui peuvent être diminuées (DELWAULLE, 1975). C'est dans le contexte de l'étude de la vitesse de « cicatrisation » d'un écosystème dégradé (TOUTAIN et al., 1983) que ce travail a été initié, en vue de déterminer l'effet dans le temps de la mise en défens sur la dynamique de reconstitution des espaces sylvo-pastoraux sénégalais du Sud du Bassin arachidier.

\section{Matériels et méthodes}

\section{Zone d'étude}

C'est dans la région de Kaolack que l'étude a été menée à bien, entre $14^{\circ} 30^{\prime}$ et $16^{\circ} 30^{\prime}$ de longitude Ouest et $13^{\circ} 30^{\prime}$ et $14^{\circ} 30^{\prime}$ de latitude Nord (FALL et al., 2008) au Sud du Bassin arachidier, au niveau des villages de Daroukoudouss (communauté rurale de Paoskoto), de Pacca Thiare Secco (communauté rurale de Thiaré) et de Keur Niène Sérère (communauté rurale de Ndiédieng). Le choix de ces villages résulte de l'existence d'un espace sylvo-pastoral en début de préservation (Daroukoudouss), d'un second avec 5 ans de mise en défens (Keur Niène Sérère) et d'un dernier vieux de 12 ans (Pacca Thiare Secco). Le climat est tropical sec, de type soudano-sahélien avec une alternance de saisons. La saison des pluies couvre les mois de juin à octobre, soit environ cinq mois ; la période sèche, de novembre à mai, est la plus longue durant sept mois. Enfin, la pluviosité annuelle varie entre 500 et $700 \mathrm{~mm}$. Les sols rencontrés dans la région sont de trois types : les sols dior, deck et deck-dior (FAYE, 2010). Les sols dior, sableux, qui représentent 30 à $80 \%$ des terres cultivables, sont réservés essentiellement à la culture de l'arachide et du mil. Les sols deck (argileux), qui sont aussi des terres cultivables, représentent 10 à $30 \%$ des terres cultivables et sont destinés à la culture du maïs, du riz et au maraîchage. Les sols deck-dior sont argilo-sableux, peu utilisés pour la plupart des cultures. Enfin, il y a des sols halomorphes, ou «tans », qui, à

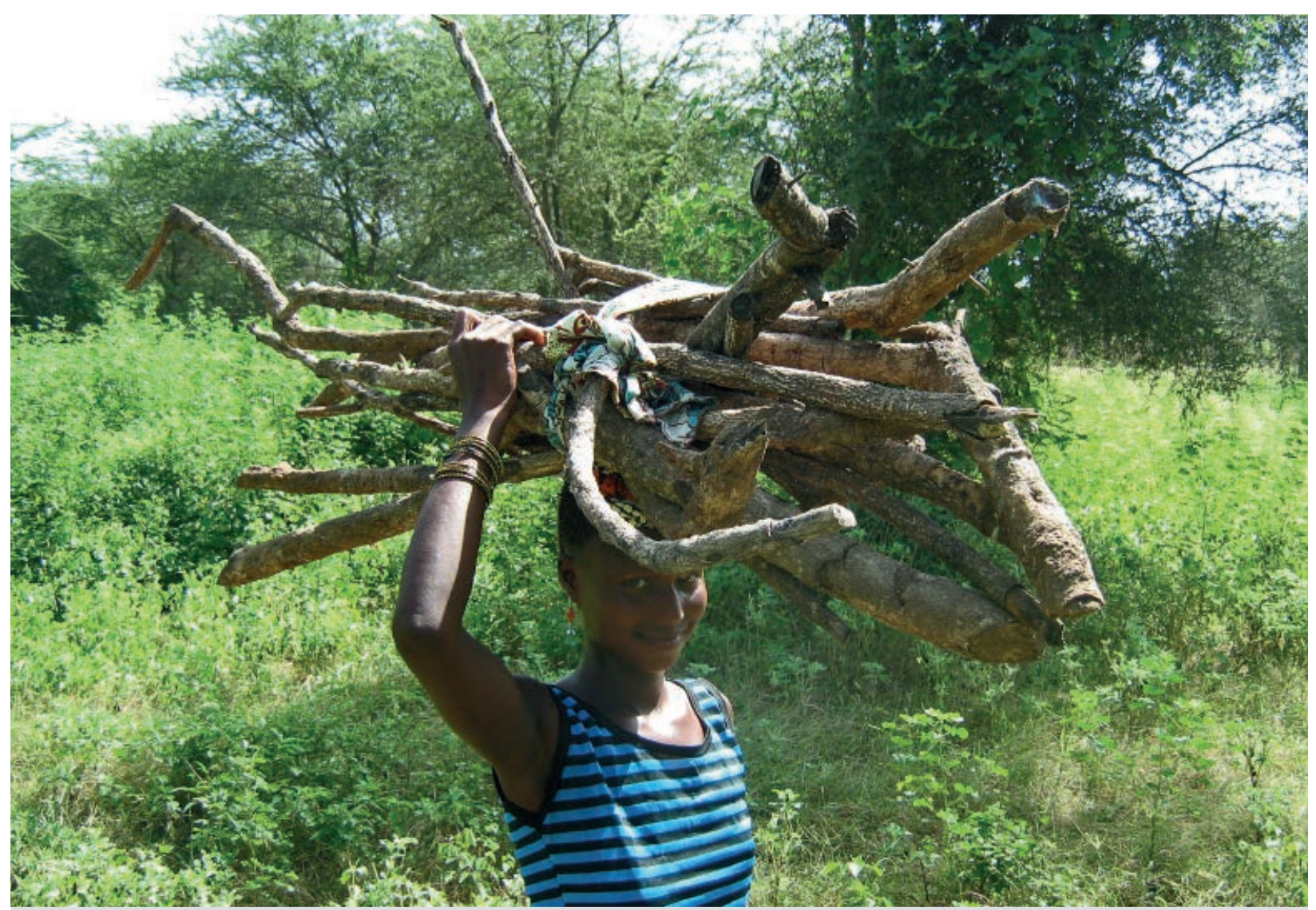

Photo 2.

Jeune fille transportant du bois issu de la mise en défens.

Photo M. Badji. 
cause de leur salinité et de leur hydromorphie, sont peu favorables à l'agriculture et se trouvent fréquemment dans les vallées, certaines espèces halophytes s'y développant. Quatre types de formations végétales caractérisent la région de Kaolack, ce sont : la savane (arbustive à arborée), la steppe arborée, la mangrove et les îlots de forêt. L'agriculture, l'élevage, l'exploitation des produits forestiers et le petit commerce sont les principales activités socio-économiques. L'agriculture concerne surtout l'arachide (Bassin arachidier) et le mil. Depuis 2003, la culture du maïs et du niébé est encouragée pour la diversification des produits vivriers. L'élevage concerne les bovins, ovins et caprins. Le cheval et l'âne sont utilisés pour la culture, le transport urbain de marchandises et de produits agricoles. Quant à l'exploitation du bois de chauffe et des produits forestiers non ligneux, elle est surtout pratiquée par les femmes et les enfants (MOHAMED MAHAMOUD et al., 2008). Il s'agit de la vente en bordure des routes et sur les marchés des fruits locaux (tamarin, jujube...).

\section{Collecte de données, placettes d'étude}

Les données pour évaluer l'impact de l'âge de la mise en défens ont été obtenues par inventaires forestiers dans des mises en défens de 1 an, 5 ans et 12 ans. Les unités d'observation sont des placettes de $900 \mathrm{~m}^{2}(30 \mathrm{~m} \times 30 \mathrm{~m})$ délimitées le long des transects à des intervalles réguliers de $50 \mathrm{~m}$. L'inventaire a été effectué sur 127 placettes, soit 37 dans la mise en défens de 1 an, 68 dans celle de 5 ans et 22 dans celle de 12 ans. Les mesures effectuées portent sur les caractéristiques dendrométriques (diamètre à $30 \mathrm{~cm}$ du sol, hauteur totale) de tous les arbres présents dans les placettes. Le potentiel de régénération des mises en défens a été apprécié par le comptage des individus dont le diamètre est inférieur à $3,5 \mathrm{~cm}$, soit $10 \mathrm{~cm}$ de circonférence selon SOKPON et al. (2006).

\section{Analyse et traitement des données}

Les paramètres structuraux retenus pour caractériser les peuplements dans les mises en défens étudiées sont la densité, le diamètre moyen, la surface terrière et la hauteur du fût. La densité d'un peuplement ligneux exprime le nombre total d'arbres par unité de surface (hectare). La surface terrière (en $\mathrm{m}^{2} / \mathrm{ha}$ ) a été calculée suivant la relation :

$G=\pi d^{2} / 4$

avec $\mathrm{G}$ en $\mathrm{m}^{2} /$ ha et $\mathrm{d}$ exprimant le diamètre à $0,3 \mathrm{~m}$ de hauteur.

La comparaison de ces caractéristiques a été effectuée par le test Anova pour évaluer la signification des différences entre les trois zones avec le programme Xlstat. Les différentes structures (structure en diamètre et répartition par classe de hauteur) ont permis d'établir l'abondance relative des arbres par classe de diamètre et de hauteur. La densité de régénération est estimée et l'importance relative des espèces dominantes est exprimée en pourcentage pour les trois milieux considérés.

\section{Résultats}

\section{Composition de la végétation ligneuse des mises en défens}

Le tableau I présente la liste des familles les plus fréquentes dans les différentes mises en défens. Soixanteneuf espèces ligneuses ont été recensées (annexe 1) dans les mises en défens du Sud du Bassin arachidier qui appartiennent à 31 familles, dont 51 espèces dans les mises en défens de 5 ans et 12 ans et 27 espèces dans celle de 1 an. Le nombre d'espèces par relevé est de 18,45 espèces dans la mise en défens de 12 ans, de 15,35 espèces dans celle de 5 ans et de 5,35 espèces dans celle de 1 an. La physionomie de la végétation est marquée par les Combretaceae $(95,04 \%)$ dans la mise en défens de 1 an, les Mimosaceae $(24,17 \%)$, les Combretaceae $(22,84 \%)$, les Rubiaceae $(21,82 \%)$, les Lamiaceae $(20,41 \%)$ dans la mise en défens de 5 ans et les Combretaceae $(47,46 \%)$, les Rubiaceae $(21,26 \%)$, les Tiliaceae $(7,75 \%)$, les Anacardiaceae $(5,51 \%)$ et les Caesalpiniaceae $(4,26 \%)$ dans la mise en défens de 12 ans. Dans l'ensemble des mises en défens, on constate que ce sont les familles des Combretaceae, des Rubiaceae, des Mimosaceae qui dominent.

\section{Caractéristiques dendrométriques des mises en défens}

L'analyse des paramètres dendrométriques montre des valeurs différentes en passant d'une mise en défens de 1 an à une mise en défens de 12 ans (tableau II). La densité des arbres est plus importante dans la mise en défens de 12 ans que dans les mises en défens de 5 ans et 1 an et est évaluée respectivement à $675 \pm 39,59$ arbres/ha, à $544 \pm$ $37,65 \mathrm{arbres} / \mathrm{h}$ a et à $19 \pm 2,66 \mathrm{arbres} / \mathrm{ha}$. Le diamètre moyen est évalué à $5,32 \mathrm{~cm}$ dans la mise en défens de $1 \mathrm{an}$, à $7,16 \mathrm{~cm}$ dans la mise en défens de 5 ans et à $7,19 \mathrm{~cm}$ dans la mise en défens de 12 ans. L'analyse statistique
Tableau I.
Liste des familles les plus fréquentes en fonction de l'âge de la mise en défens.

\begin{tabular}{lcccr} 
Familles & \multicolumn{4}{c}{ Âge de la mise en défens } \\
& $\mathbf{1}$ an & $\mathbf{5}$ ans & $\mathbf{1 2}$ ans \\
Anacardiaceae & - & & \\
\hline Apocyniaceae & - & - & $5,51 \%$ \\
\hline Bignoniaceae & - & $1,42 \%$ & $2,01 \%$ \\
\hline Caesalpiniaceae & - & $2,41 \%$ & $4,26 \%$ \\
\hline Celastraceae & - & - & $1,49 \%$ \\
\hline Combretaceae & $95,04 \%$ & $22,84 \%$ & $47,46 \%$ \\
\hline Ebenaceae & - & - & $3,74 \%$ \\
\hline Lamiaceae & - & $20,41 \%$ & - \\
\hline Mimosaceae & $1,02 \%$ & $24,17 \%$ & $1,19 \%$ \\
\hline Rubiaceae & $2,23 \%$ & $21,82 \%$ & $21,26 \%$ \\
\hline Tiliaceae & - & - & $7,75 \%$ \\
\hline Autres familles & $1,70 \%$ & $6,90 \%$ & $3,15 \%$ \\
\hline & & & \\
\hline
\end{tabular}


Annexe I.

Liste des familles, genres et espèces inventoriés dans les différents espaces sylvo-pastoraux du Sud du Bassin arachidier ( $\mathrm{x}=$ présence).

Familles Espèces

Anacardiaceae

Annonaceae

Apocynaceae

Asclepiadaceae

Balanitaceae

Bignoniaceae

Bombacaceae

Burseraceae

Caesalpiniaceae

Capparaceae

Celastraceae

Combretaceae

Ebenaceae

Euphorbiaceae

Fabaceae

Hymenocardiaceae

Lamiaceae

Loganiaceae

Meliaceae

Mimosaceae

Olacaceae

Opiliaceae

Polygolaceae

Rhamnaceae

Rubiaceae

Sterculiaceae

Tiliaceae

Verbenaceae

Vitaceae
Ozoroa insignis Del.

Lannea acida A. Rich.

Lannea velutina A. Rich.

Sclerocarya birrea (A. Rich.) Hochst.

Hexalobus monopetalus (A. Rich.) Engl. et Diels

Strophantus sarmentosus DC. Incl.

Baissea multiflora A. DC.

Leptadenia hastata (Pers.) Decne.

Balanites aegyptiaca (L.) Del.

Stereospermum kunthianum Cham.

Bombax costatum Pellegr. et Vuillet

Adansonia digitata $\mathrm{L}$.

Commiphora africana (A. Rich.) Engl.

Cassia sieberiana DC.

Cordyla pinnata (Lepr. ex A. Rich.) Milne-Redhead

Piliostigma reticulatum (DC.) Hochst.

Tamarindus indica L.

Detarium microcarpum Gill. et Perr.

Cadaba farinosa Forssk.

Caparis tomentosa Lam.

Maerua angolensis DC.

Maytenus senegalensis (Lam.) Exell

Anogeissus leiocarpus (DC) Gill. et Perr.

Combretum glutinosum Perr. ex DC

Combretum leucardii Engl. et Diels

Combretum aculeatum Vent.

Combretum nigricans Lepr. ex Guill. et Perr.

Guiera senegalensis J.F. Gmel.

Terminalia macroptera Guill. et Perr.

Diospyros mespiliformisHochst. ex A. Rich.

Jatropha gossypiifolia L.

Flueggea virosa (Roxb. ex Willd.) Voigt

Euphorbia balsamifera Ait.

Pterocarpus erinaceus Poir.

Lonchocarpus laxiflorus Guill. et Perr

Hymenocardia acida Tul.

Hoslundia opposita Vahl

Strychnos spinosa Lam.

Azadirachta indica A. Juss.

Acacia raddiana Savi

Dichrostachys cinerea (L.) Wight et Aren.

Acacia macrostachya Reichenb. ex. DC.

Acacia senegal (L.) Willd.

Acacia seyal Del.

Albizzia chevalieri Harms

Antiaris africana Engl.

Ficus iteophylla Miq.

Ficus capensis Thunb.

Ximenia americana L.

Opilia celtidifolia (Guill. et Perr.) Endl. ex Walp.

Securidaca longepedunculata Fres.

Ziziphus mucronata Willd.

Ziziphus mauritiana Lam.

Feretia apodanthera Del.

Gardenia erubescens Stapf et Hutch.

Gardenia ternifolia Schumach. et Thonn.

Crossopteryx febriguga (Afzel. ex G. Don) Benth.

Mitragyna inermis (Willd.) Kuntze

Solanum uncanum L.

Sterculia setigera Del.

Grewia bicolor Juss.

Grewia Flavescens Juss.

Grewia lasiodiscus K. Shum.

\section{Âge des mises en défens \\ 5 ans \\ 12 ans}

\section{$x$}

$x$

$x \quad x$

$\begin{array}{ll}\mathrm{x} & \mathrm{x} \\ \mathrm{x} & \mathrm{x} \\ \mathrm{x} & \mathrm{x}\end{array}$

$\begin{array}{lll}x & x & x \\ x & x\end{array}$

$x$

$x$

$\mathrm{x}$

$\begin{array}{lll}x & x & x \\ x & x & x\end{array}$

$x$
$x$
$x$
$x$
$x$

$\mathrm{x}$

Vitex madiensis Oliv.

Lippia chevalieri Moldenk

Cissus populnea Guill. et Perr.

Cissus cymosa Schum. et Thonn.

Cissus adenocaulis Steud

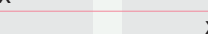

$x \quad x$

$x \quad x$

$x$

$\begin{array}{ll}x \\ x & x \\ x & x\end{array}$

$\begin{array}{ccc}x & x & x \\ x & x & x\end{array}$

$\mathrm{x}$

$x$

$x$
$x$
$x$
$x$
$x$
$x$
$x$
$x$
$x$
$x$
$x$
$x$
$x$
$x$
$x$
$x$
$x$
$x$
$x$
$x$
$x$
$x$
$x$
$x$

$x$
$x$
$x$
$x$
$x$
$x$
$x$
$x$
$x$
$x$
$x$
$x$
$x$
$x$
$x$
$x$
$x$
$x$
$x$
$x$
$x$
$x$
$x$
$x$

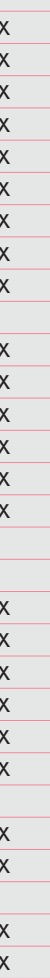

$x$

$\mathrm{x}$

$\mathrm{x}$

$x$

$x$
$x$
$x$
$x$
$x$
$x$
$x$
$x$
$x$
$x$
$x$
$x$
$x$
$x$
$x$
$x$
$x$
$x$
$x$
$x$
$x$
$x$
$x$
$x$

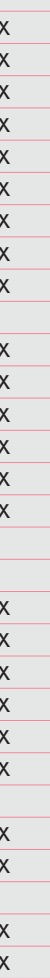

$x$
$x$
$x$
$x$
$x$
$x$
$x$
$x$
$x$
$x$
$x$
$x$
$x$
$x$
$x$
$x$
$x$
$x$
$x$
$x$
$x$
$x$
$x$
$x$

$\mathrm{X}$

$x-x$

$x$

$\mathrm{x}$

$x \quad x$

$x$

$\mathrm{x}$

X

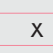

X $x$

$\begin{array}{lll} & x & x \\ x & x & x\end{array}$

X

$\mathrm{x}$

$\mathrm{x}$

$\begin{array}{ll}x & x \\ x & x\end{array}$

$x$

$\mathrm{x}$

$\mathrm{x}$

$\mathrm{x}$

$\mathrm{x}$

X

X

$\begin{array}{lll}x & x \\ x & x\end{array}$

$x+x$

$\mathrm{X}$

$\mathrm{x}$

$x$

$x$

$x$

$\mathrm{x}$

$\begin{array}{ll}x & x \\ x & x\end{array}$

$x-\frac{x}{x}$

$\begin{array}{ll}\mathrm{x} & \mathrm{x} \\ \mathrm{x} & \mathrm{x}\end{array}$

$x$

\begin{tabular}{|l|l|}
\hline$x$ & $x$ \\
\hline$x$ & $x$ \\
\hline$x$ & \\
\hline
\end{tabular}


Tableau II.

Principales caractéristiques dendrométriques des mises en défens.

\begin{tabular}{|l|c|c|c|}
\hline Paramètres dendrométriques & $\mathbf{1}$ an & $\mathbf{5}$ ans & $\mathbf{1 2}$ ans \\
\hline Densité $(\mathrm{N} / \mathrm{ha})$ & $19 \pm 2,66$ & & \\
\hline Diamètre moyen $(\mathrm{cm})$ & $5,32 \pm 6,87$ & $7,16 \pm 5,01$ & $7,19 \pm 39,59$ \\
\hline Hauteur moyenne $(\mathrm{m})$ & $2,46 \pm 1,07$ & $3,95 \pm 1,33$ & $4,22 \pm 1,97$ \\
\hline Surface terrière $\left(\mathrm{m}^{2} / \mathrm{ha}\right)$ & $0,11 \pm 0,02$ & $3,22 \pm 0,27$ & $3,78 \pm 0,22$ \\
\hline
\end{tabular}

Tableau III.

État de la régénération dans les différentes mises en défens.

\begin{tabular}{l|c|c|c|} 
& $\mathbf{1}$ an & $\mathbf{5}$ ans & $\mathbf{1 2}$ ans \\
Densité régénération & & & \\
\hline Combretum glutinosum Perr. ex DC. & $206(100 \%)$ & $2187(100 \%)$ & $2289(100 \%)$ \\
\hline Guiera senegalensis J.F. Gmel. & $919(28,66 \%)$ & $290(13,28 \%)$ & $834(36,43 \%)$ \\
\hline Feretia apodanthera Del. & $2087(65,09 \%)$ & $17(0,8 \%)$ & $70(3,04 \%)$ \\
\hline Acacia seyal Del. & $71(2,22 \%)$ & $524(23,95 \%)$ & $517(22,57 \%)$ \\
\hline Anogeissus leiocarpus (DC.) Gill. et Perr. & $0(0)$ & $385(17,59 \%)$ & $5(0,22 \%)$ \\
\hline Hoslundia opposita Vahl & $1(0,03 \%)$ & $43(1,96 \%)$ & $65(2,87 \%)$ \\
\hline Grewia bicolor Juss. & $0(0)$ & $581(26,58 \%)$ & $3(0,15 \%)$ \\
\hline
\end{tabular}

révèle une différence significative pour le diamètre moyen entre les mises en défens de 1 an et 5 ans $(P=0,003)$ et entre les mises en défens de 1 an et 12 ans $(P=0,003)$. Par contre, il n'y a pas de différence significative pour le diamètre moyen entre les mises en défens de 5 ans et 12 ans $(P=$ 0,852). La hauteur des arbres est évaluée à 2,46 $\pm 1,07 \mathrm{~m}$ dans la mise en défens de 1 an, à 3,95 $\pm 1,33 \mathrm{~m}$ dans la mise en défens de 5 ans et à 4,22 $\pm 1,97$ m dans la mise en défens de 12 ans. Le test de variance effectué sur les valeurs obtenues pour ce paramètre traduit une différence significative de la hauteur moyenne entre les mises en défens de 1 an et 5 ans ( $P<0,0001$ ), entre les mises en défens de 1 an et 12 ans $(P<0,0001)$ et entre les mises en défens de 5 ans et 12 ans $(P=0,000)$. La surface terrière très faible dans la mise en défens de 1 an $(0,11 \pm$ $0,02 \mathrm{~m}^{2} / \mathrm{ha}$ ) est beaucoup plus importante dans les mises en défens de 5 ans $\left(3,22 \pm 0,27 \mathrm{~m}^{2} / \mathrm{ha}\right)$ et 12 ans $(3,78 \pm$ $\left.0,22 \mathrm{~m}^{2} / \mathrm{ha}\right)$.

\section{Impacts de l'âge de la mise en défens sur la dynamique des peuplements}

La distribution des arbres par classes de diamètre montre une distribution en « $L$ » avec une prédominance des individus de petit diamètre traduisant une dynamique régulière du peuplement des mises en défens de 1 an et 5 ans (figure $1 \mathrm{a}, 1 \mathrm{~b}$ ). La distribution de la surface terrière montre que, dans la mise en défens de 1 an, les arbres de classes de diamètre de $[3,5-5 \mathrm{~cm}],[15-20 \mathrm{~cm}]$ et $[35-40 \mathrm{~cm}]$ cumulent l'essentiel de la surface terrière qui est très faible. Par contre, la structure diamétrique des individus recensés dans la mise en défens de 12 ans montre une distribution en cloche dissymétrique (figure $1 \mathrm{c}$ ). Cette répartition fait apparaître une prédominance des individus de diamètre compris entre 5 et $10 \mathrm{~cm}$. La répartition de la surface terrière indique que la classe de diamètre $5-10 \mathrm{~cm}$ présente la surface terrière la plus élevée dans les mises en défens de 5 ans et 12 ans.

\section{Impacts de l'âge de la mise en défens sur la régénération}

Le comptage de la régénération (tableau III) montre une densité plus importante dans la mise en défens de 1 an (3 206 tiges/ha) que dans les mises en défens de 5 ans et 12 ans. En considérant les espèces dominantes, Combretum glutinosum (919 tiges/ha) et Guiera senegalensis (2 087 tiges/ha) régénèrent plus dans la mise en défens de 1 an. En revanche, Hoslandia opposita (581 tiges/ha), Feretia apodanthera (524 tiges/ha), Combretum glutinosum (290 tiges/ha) et Acacia seyal (385 tiges/ha) produisent plus de régénération dans la mise en défens de 5 an ; dans celle de 12 ans, ce sont Combretum glutinosum (834 tiges/ha), Feretia apodanthera (517 tiges/ha) et Grevia bicolor (133 tiges/ha). 


\section{Discussion et conclusion}

L'objectif de ce travail a été de déterminer l'effet de l'âge de la mise en défens sur la dynamique de reconstitution de la végétation ligneuse des espaces sylvo-pastoraux du Sud du Bassin arachidier du Sénégal. II ressort des résultats de l'inventaire que la flore est riche de 69 espèces ligneuses appartenant à 31 familles. Ces familles sont dominées par les Combretaceae, les Rubiaceae, les Mimosaceae. Selon BA et NOBA (2001), ces familles sont classées parmi les plus importantes de la flore vasculaire du Sénégal. Le nombre moyen d'espèces par relevé et le nombre total d'espèces sont plus élevés dans les mises en défens de 5 ans et 12 ans, ce qui s'explique par le retour des espèces les plus sensibles à la forte pression anthropique pour le bois ou le fourrage (Strophantus sarmentosus, Pterocarpus erinaceus, Bombax costatum, Sterculia setigera, Lannea acida, Detarium microcarpum, Maerua angolensis, Commiphora africana, Cordyla pinnata, Grewia lasiodiscus, Hexalobus monopetalus, Adansonia digitata). Ce résultat corrobore celui trouvé par GROUZIS (1991), DIATTA (1994), ALBERGEL et al. (1995),

DIATTA et FAYE (1996) et MBAYE et al. (2009) qui ont montré que la mise en défens permet une augmentation du nombre d'espèces ligneuses par rapport à la situation de départ. YOSSI (1995) trouve que le nombre d'espèces augmente en fonction de l'âge ou de la durée d'abandon de la culture dans les jachères d'âges différents. La diversité floristique des mises en défens de 5 ans et 12 ans a été comparée à celle de la forêt de Welor et de Sambandé (46 espèces) (SAMBOU et al., 2008 ; MOHAMED MAHAMOUD et al., 2008) et celle des terroirs villageois de Latmingué (FAYE et al., 2008) qui se trouvent dans la même région. La forte diversité de ces mises en défens résulte d'un accompagnement de conventions locales interdisant toute forme d'intervention humaine pour favoriser la régénération naturelle.

La densité des individus et la surface terrière augmentent en fonction de l'âge de la mise en défens. Les faibles valeurs de densité et de surface terrière obtenues dans la mise en défens de 1 an peuvent s'expliquer par la forte anthropisation pour le bois de chauffe qu'a subie cette mise en défens avant la protection. Ce résultat corrobore celui trouvé par SOUNON BOUKO et al., 2007, qui ont montré une augmentation de la densité des individus et de la surface terrière en fonction de l'âge (passage des jachères aux friches). Sur le plan structural, la distribution des arbres par classes de diamètre montre une distribution en « $L$ » avec
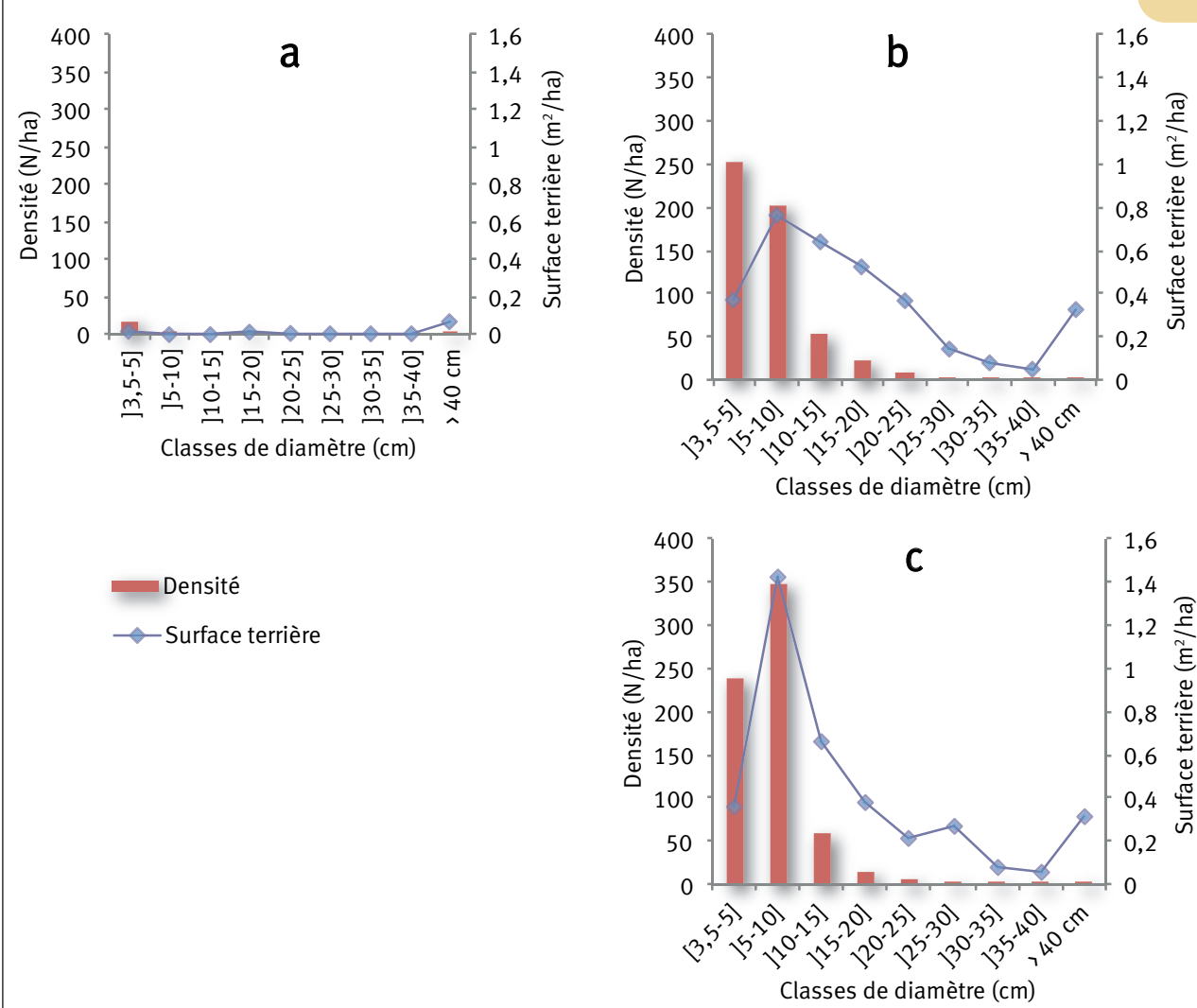

Figure 1.

Structure des classes de diamètre des arbres dans les mises en défens de 1 an (a), 5 ans (b) et 12 ans (c).

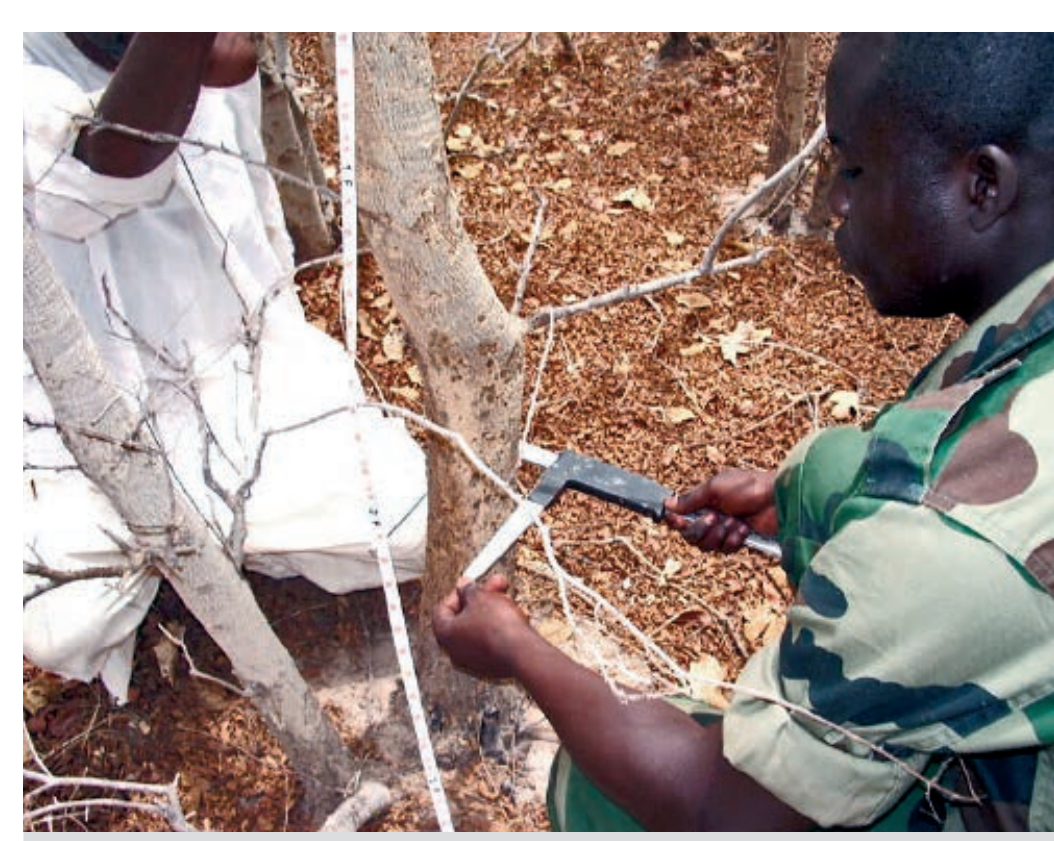

Photo 3.

Mesure du diamètre à $0,30 \mathrm{~m}$ avec un compas forestier. Photo M. Badji. 
la tige ou de racines dépend de plusieurs facteurs dont entre autres un stress (labour, feu...) et peut généralement être induite par les perturbations anthropiques. Ce qui n'est pas le cas dans ces mises en défens où les coupes sont interdites.

En guise de conclusion, il est possible d'avancer que cette première étude en appelle d'autres devant bénéficier du recul dans le temps. Elle a surtout mis en évidence et quantifié l'effet de l'âge de mise en défens sur la reconstitution des espaces sylvo-pastoraux du Sud du Bassin arachidier. Elle a montré qu'une simple protection de quelques années a permis d'aboutir dès cinq ans à l'amélioration des caractéristiques structurales et fonctionnelles en augmentant le nombre d'espèces, la densité, la surface terrière. La structure du peuplement révèle la prédominance des individus de diamètre inférieur à $10 \mathrm{~cm}$, traduisant une bonne régénération des espèces qui se multiplient par semis.

\section{Remerciements}

Ce travail a été possible grâce au financement du projet « Renforcement des stratégies locales de gestion des espaces sylvo-pastoraux intervillageois du Bassin arachidier (Sénégal) », financé par le Crdi.

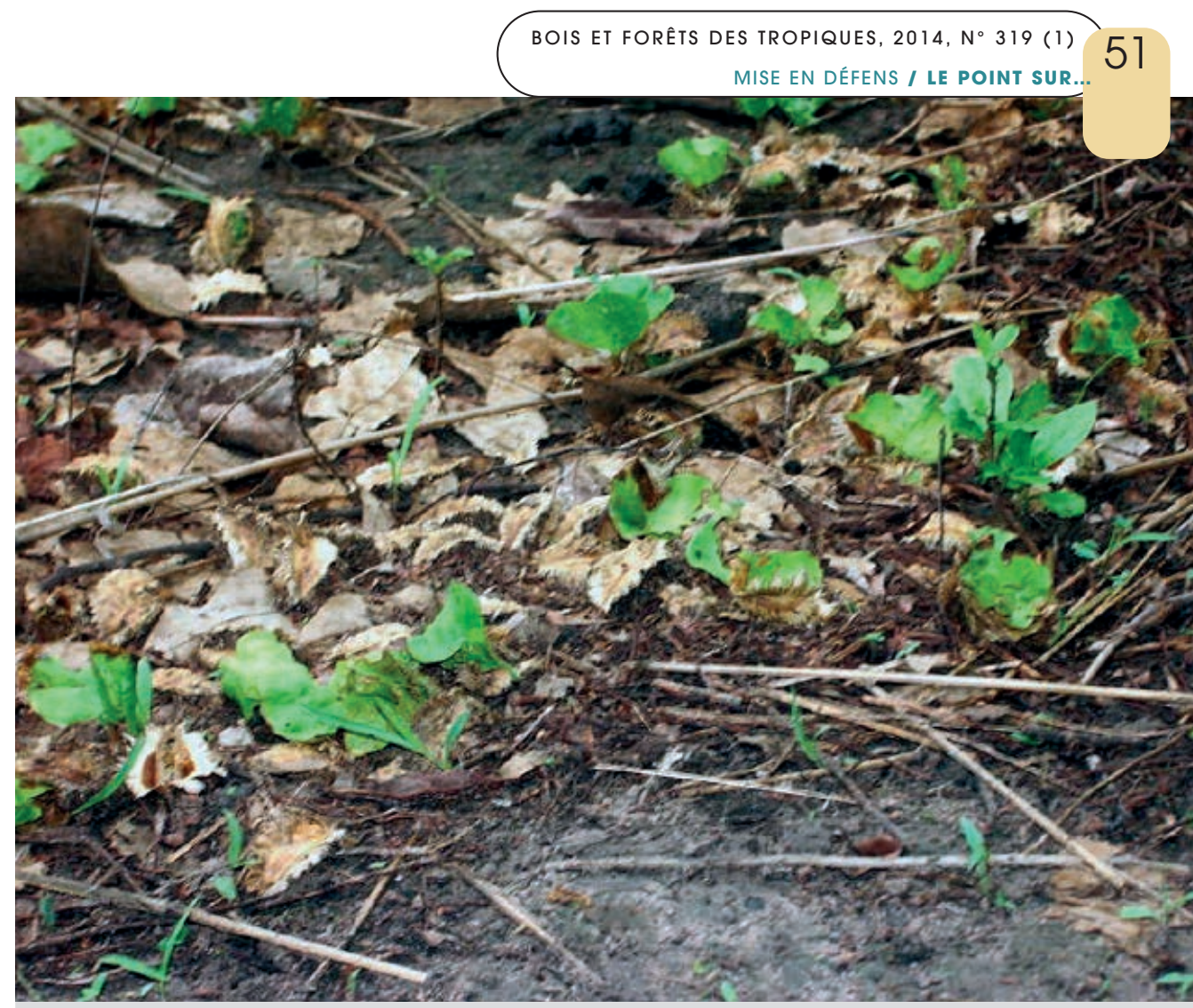

Photo 6.

Régénération de Combretum glutinosum.

Photo M. Badji.

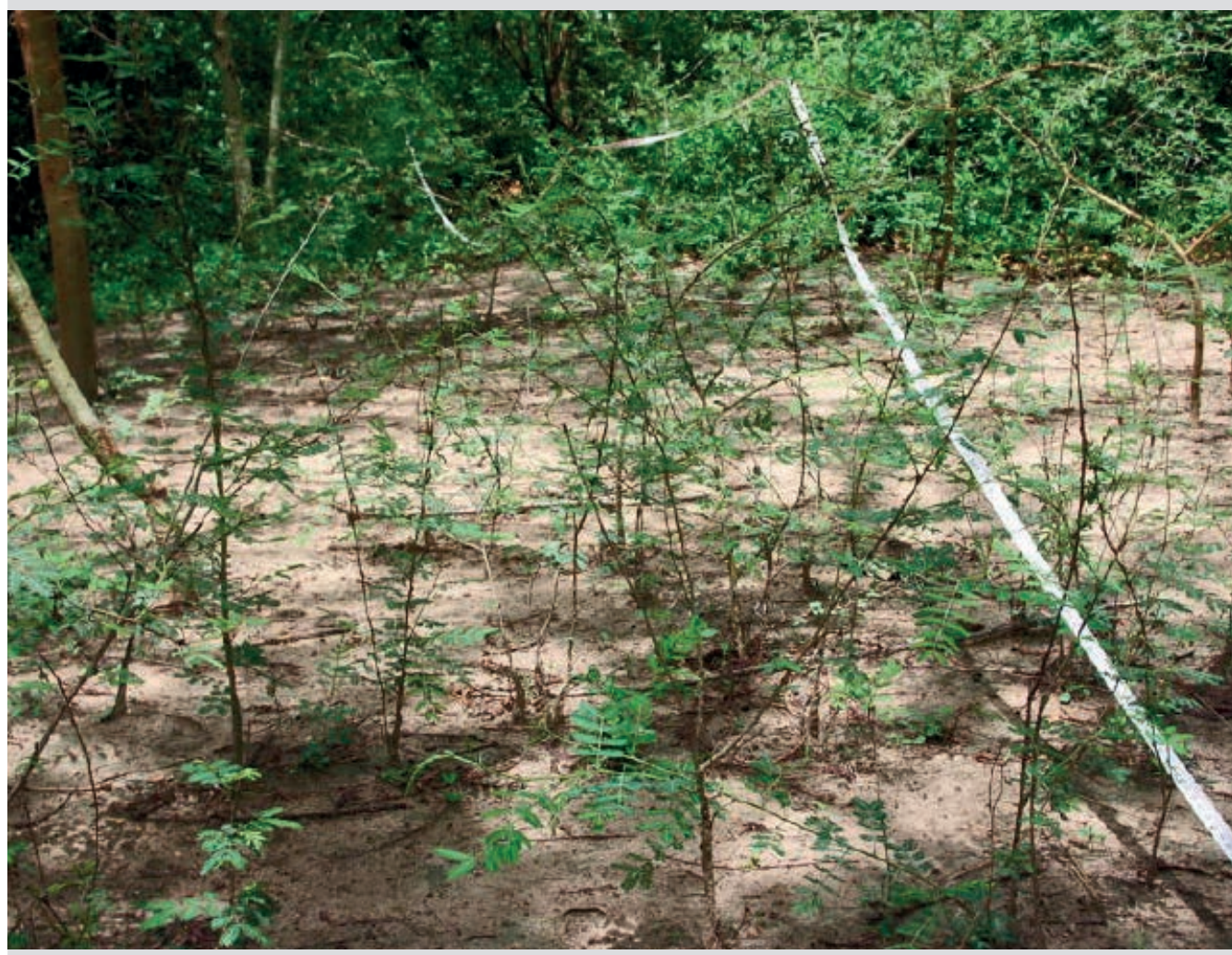

Photo 7.

Régénération de Acacia seyal.

Photo M. Badji. 


\section{Références bibliographiques}

AKPO L. E., GASTON A., GROUZIS M., 1995. Structure spécifique d'une végétation sahélienne. Cas de Wiidu Thiengoli (Ferlo, Sénégal). Bulletin du Muséum National d'Histoire Naturelle, section B, Adansonia, 17 (1-2) : 39-52.

AKPO L. E., GROUZIS M., 1996. Influence du couvert sur la régénération de quelques espèces ligneuses sahéliennes (NordSénégal, Afrique occidentale). Webbia, 50 (2) : 247-263.

ALBERGEL J., DIATTA M., GROUZIS M., PÉREZ P., SĖNE M., 1995. Réhabilitation d’un écosystème semi-aride au Sénégal par l'aménagement des éléments du paysage. In : Pontanier R., M'Hiri A., Akrimi N., Aronson J., Le Floc'h E. (éds). L'homme peut-il refaire ce qu'il a défait? Montrouge, France, John Libbey Eurotext, 293-306.

BA A. T., NOBA K., 2001. Flore et biodiversité végétale au Sénégal. Sécheresse, 12 (3) : 149-155.

BAZILE D., 1998. La gestion des espèces ligneuses dans l'approvisionnement en énergie des populations. Cas de la zone soudanienne du Mali. Thèse de doctorat, Université de Toulouse Le Mirail, France, $335 \mathrm{p}$.

DELWAULLE J.-C., 1975. Le rôle du forestier dans l'aménagement du Sahel. Bois et Forêts des Tropiques, 60 : 3-22.

DEMBELE F., PICARD N., KAREMBÉ M., BIRNBAUM P., 2007. Tree vegetation patterns along a gradient of human disturbance in the sahelian area of Mali. Journal of Arid Environment, 64 : 284-97.

DIATTA M., 1994. Mise en défens et techniques agroforestières au Sine Saloum (Sénégal). Effets sur la conservation de l'eau, du sol et sur la production primaire. Thèse de doctorat de l'Université scientifique L. Pasteur (Strasbourg I), Géographie physique, 202 p.

DIATTA M., FAYE E. H., 1996. Effets de quelques années de protection sur la jachère en zone sahélo-soudanienne du Sénégal : structure et production primaire. In : Floret C. (éd.). La jachère, un lieu de production. Dakar, Sénégal, Orstom, 33-41.

FALL B., NDAO M., NDIAYE M., NDIAYE C., DIOUF P., YOUSSIFI F., 2008. Situation économique et sociale de la région de Kaolack 2008. Dakar, Sénégal, Agence nationale de la statistique et de la démographie, $158 \mathrm{p}$.

FAYE E., DIATTA M., SAMBA A. N. S., LEJOLY J., 2008. Usages et dynamique de la flore ligneuse dans le terroir villageois de Latmingué (Sénégal). Journal des Sciences et Technologies, $7:$ 43-58.

FAYE E. H., 2010. Diagnostic partiel de la flore et de la végétation des Niayes et du Bassin arachidier au Sénégal : application de méthodes floristique, phytosociologique, ethnobotanique et cartographique. Thèse Université libre de Bruxelles, Faculté des sciences, École interfacultaire de bioingénieurs, Service d'écologie du paysage et systèmes de production végétale, Belgique, $266 \mathrm{p}$.

GROUZIS M., 1988. Structure, productivité et dynamique des systèmes écologiques sahéliens (Mare d'Oursi, Burkina Faso). Paris, France, Orstom, coll. Études et Thèses, 336 p.
GROUZIS M., 1991. Évolution et seuils de rupture des systèmes écologiques. In : Claude J., Grouzis M., Milleville P. (éds). Un espace sahélien : la mare d’Oursi, Burkina Faso. Paris, France, Orstom, 126-137.

MBAYE S. M., SECK M. L., FALL M., DIOP S. S., TOUNKARA A., 2009. Les conventions locales au Sénégal, Mbédap : à l'épreuve du temps. Dakar, Sénégal, led Afrique, Conventions locales $\mathrm{n}^{\circ} 5,25 \mathrm{p}$.

MOHAMED MAHAMOUD C., LO M., BASSĖNE E., AKPO L. E., 2008. Caractéristiques de la flore et de la végétation ligneuses de forêts communautaires de la zone soudanosahélienne au Sénégal. Journal des Sciences et Technologies, 6 (2) : 72-85.

NOUVELLET Y., 1992. Évolution d'un taillis de formation naturelle en zone soudanienne du Burkina Faso. Thèse Université Pierre et Marie Curie, Paris VI, France, 356 p.

PELTIER R., LAWALI E. M., MONTAGNE P., 1994. Aménagement villageois des brousses tachetées au Niger. Première partie. Le milieu : potentiel et contraintes. Bois et Forêts des Tropiques, $242:$ 59-76.

SAMBOU B., GOUDIABY A., MADDSEN J. E., BA A. T., 1994. Étude comparative des modifications de la flore et de la végétation ligneuses dans les forêts classées de Koutal et de l'île Kouyong (Centre-Ouest du Sénégal). Journal d'Agriculture Traditionnelle et de Botanique Appliquée, nouvelle série, 36 (1) : 87-100.

SAMBOU B., BÂ A. T., MBOW C., GOUDIABY A., 2008. Studies of the Woody Vegetation of the Welor Forest Reserve (Senegal) for Sustainable Use. West African Journal of Applied Ecology, 13 (1), 10 p.

SOKPON N., BIAOU S. H., OUINSAVI C., HUNHYET O., 2006. Bases techniques pour une gestion durable des forêts claires du Nord-Bénin : rotation, diamètre minimal d'exploitabilité et régénération. Bois et Forêts des Tropiques, 287 : 45-57.

SOUNON BOUKO B., SINSIN B., GOURA SOULÉ B., 2007. Effets de la dynamique d'occupation du sol sur la structure et la diversité floristique des forêts claires et savanes au Bénin. Tropicultura, 25 (4) : 221-227.

TOUTAIN B., BORTOLI L., DULIEU D., FORIGIRNI G., MENAUT J.-C., PIOT J., 1983. Espèces ligneuses et herbacées dans les écosystèmes pâturés sahéliens de Haute-Volta. Synthèse des résultats du programme Acc-Griza (Lat), Gerdat, $124 \mathrm{p}$.

YOSSI H., 1995. Dynamique de la végétation post-culturale en zone soudanienne au Mali. Thèse Institut supérieur de formation et de recherche appliquée (Isfra), Bamako, Mali, 170 p. 